\title{
Prevalencia de dislexia del desarrollo en estudiantes panameños
}

\author{
Daniel Cubilla-Bonnetier ${ }^{1, *}$, Abdel Solís-Rodríguez ${ }^{1}$, \\ Francisco Farnum ${ }^{2}$, Jessibeth Caballero ${ }^{3}$ \\ ${ }^{1}$ Dirección de Investigación y Desarrollo, Universidad Católica Santa María la Antigua \\ ${ }^{2}$ Centro Regional Universitario de Colón, Universidad de Panamá \\ ${ }^{3}$ Instituto de Salud Global y Medicina Tropical, Universidad Católica Santa María la Antigua
}

*Autor para correspondencia. E-mail: dcubilla@usma.ac.pa

Recibido: 16 de octubre de 2020

Aceptado: 23 de noviembre de 2020

\begin{abstract}
Resumen
El trastorno del desarrollo del aprendizaje con dificultades en la lectura, o dislexia del desarrollo, es una dificultad de tipo neurobiológico, crónico y con un componente genético que afecta a la funcionalidad de lectura y que, por sus consecuencias escolares, emocionales y sociales, constituye un problema de salud pública que merece una especial atención sanitaria y educativa, para cuyo abordaje es imprescindible conocer el tamaño de la población afectada. Para poder llevar a cabo una estimación de la prevalencia de dislexia en la Ciudad de Panamá, se efectuó una evaluación individualizada de los niños y niñas de una muestra intencional de 216 estudiantes de $4^{\circ}$ a $6^{\circ}$ grado de Primaria pertenecientes a escuelas públicas y particulares. Se halló una prevalencia de $5.56 \%$ de dislexia, sin diferencias relevantes entre géneros y entre sistema educativo público/particular. Se relacionan los resultados con los de otros estudios similares efectuados con participantes de lengua española y se discuten las proporciones encontradas de subtipos de dislexia.
\end{abstract}

Palabras Clave: prevalencia, dislexia, trastorno específico de la lectura, género, sistema escolar.

\begin{abstract}
Developmental learning disorder with impairment in reading, or developmental dyslexia, is a neurobiological difficulty, chronical and with a genetic component, that affects reading functionality and that, because of its educational, emotional and social consequences, constitutes a public health problem that deserves special health and educational care, for whose approach it is essential to know the size of the affected population. To carry out an estimate of the prevalence of dyslexia in Panama City, an individualized assessment of children of an intentional sample of 216 students from 4th to
\end{abstract}


6th grades of Primary belonging to public and private schools was carried out. A prevalence of 5.56\% of dyslexia was found, with no relevant differences between the sexes and between the public/particular education system. The results are related to those of other similar studies conducted with Spanish language participants and the proportions found of dyslexia subtypes are discussed.

Keywords: prevalence, dyslexia, specific reading disorder, gender, education system.

\section{Introducción}

La dislexia del desarrollo (DD), o evolutiva, o trastorno del desarrollo del aprendizaje con dificultades en la lectura, por su denominación en el CIE-11 (Organización Mundial de la Salud, 2018), es una dificultad "de origen neurobiológico caracterizada por la presencia de dificultades en la precisión y fluidez en el reconocimiento de palabras y por un déficit en las habilidades de decodificación y el deletreo" (Lyon, Shaywitz y Shaywitz, 2003).

Se trata de un trastorno crónico y con un componente genético (Galaburda et al., 2006) que no suele ser detectado hasta el inicio formal del aprendizaje de la lectoescritura y que constituye hasta el $80 \%$ de todas las dificultades específicas de aprendizaje (Shaywitz, 1998). Implica dificultades en la precisión de la lectura de palabras, en la velocidad de la decodificación y frecuentemente en la calidad de la comprensión de la lectura (American Psychiatric Association, 2013). Por su sintomatología, que incluye, además de las implicaciones mencionadas, una mayor carga cognitiva durante la tarea de decodificación lectora (Martin, Kronbichler y Richlan, 2016), tiene un impacto decisivo en el desempeño académico de los niños y niñas que la padecen (Sainio, Eklund, Ahonen y Kiuru, 2019), constituyendo una de las principales causas de fracaso y abandono escolar.

La revisión de la literatura sobre estudios de prevalencia de trastorno específico de la lectura muestra que la gran disparidad de metodologías empleadas para el diagnóstico provoca importantes variaciones en los resultados de los estudios consultados (Carrillo, Alegría, Miranda y Sánchez Pérez, 2011). Otro factor que se ha identificado como responsable de la variación de las cifras de prevalencia en la literatura es la influencia del grado de transparencia/opacidad ortográfica de la lengua de lectura, constatándose habitualmente mayor número de casos en lenguas en las que la correspondencia entre grafemas y fonemas es menos unívoca y regular (Wydell, 2012), como es el caso del inglés. Si se centra el foco en los estudios publicados en países de lengua española (con una ortografía considerada de alto nivel de transparencia), las cifras de prevalencia oscilan entre el 3.2\% (Jiménez E., Guzmán, Rodríguez y Artiles, 2009) y el 11.8\% (Carrillo, Alegría, Miranda y Sánchez, 2011), ambos estudios realizados en España. Específicamente en Latinoamérica, encontramos estudios de prevalencia en los siguientes países, con diversos resultados: dos en Colombia, concretamente en Barranquilla, con 3.3\% de DD (De los Reyes Aragón et al., 2008) y en Bogotá, con 3.63\% (Pardo, 2015), en Ciudad de Guatemala, con 8\% (Jiménez y García de la Cadena, 2007) y Ecuador, con un discrepante 26.57\% (Vélez et al., 2015).

Describir la dificultad lectora de la persona requiere manejar el concepto de las rutas fonológica (o sub-léxica, o indirecta) y léxica (o directa) de lectura, que integran el modelo de la Doble Ruta (Coltheart y Rastle, 1994). Mientras que la ruta fonológica constituye la estrategia de lectura basada en la descomposición de la palabra en unidades más pequeñas a través de la conversión de sus grafemas en fonemas (para formar sílabas o morfemas y ensamblar posteriormente los componentes y acceder a la palabra), la ruta léxica permite el reconocimiento directo de la forma ortográfica de la palabra 
como un conjunto, ya presente en el léxico visual de la persona, al estar previamente familiarizada con ella. Aunque la existencia de categorías diagnósticas o subtipos de dislexia es objeto de debate, tradicionalmente se ha usado la categoría "dislexia fonológica", o "disfonética" para referirse a un déficit específico en el uso de la ruta fonológica de lectura, la de "dislexia superficial", o "visual" para referirse a un uso anómalo de la ruta léxica de lectura y, por último, la de "dislexia mixta", o "profunda" para clasificar a lectores con dificultades severas en el uso de ambas rutas (Serrano y Defior, 2004).

La alta prevalencia de la DD, junto con la importante porción de fracaso escolar que se le atribuye, el impacto sobre la autoestima de la persona que la padece (Zuppardo, Serrano y Pirrone, 2017), e incluso el aumento de la propensión a presentar conductas de abuso de sustancias y delincuencia (Alemany, 2019), justifican que sea considerado un verdadero problema de salud pública. Consecuentemente, es indispensable la puesta en marcha de políticas públicas de abordaje eficiente desde los sectores de salud y educación. Para lograrlo, es indispensable estimar previamente el tamaño de la población afectada por el trastorno para poder dotar a los futuros programas de intervención de medios humanos y materiales suficientes. El presente trabajo tiene como objetivo hacer una estimación preliminar de la prevalencia de DD en la Ciudad de Panamá, de la que no existe hasta la fecha ninguna cuantificación publicada.

\section{Materiales y Métodos}

\subsection{Diseño de estudio}

Se empleó un diseño no experimental, transversal, descriptivo de corte cuantitativo y no probabilístico.

\subsection{Muestra}

Se seleccionó de forma intencional una serie de 6 escuelas ( 3 del sistema público y 3 del particular) de la Ciudad de Panamá de ubicaciones diversas y con acudientes de origen socioeconómico diferenciado, de las que se extrajo de forma no intencional una muestra de 216 participantes (108 niños y 108 niñas) pertenecientes a $4^{\circ}(n=74), 5^{\circ}(n=72)$ y $6^{\circ}(n=70)$ grado de Educación Primaria. Los participantes con diagnósticos conocidos que pudieran interferir con el de dislexia (como trastornos generalizados del desarrollo o sensoriales) fueron descartados.

Todos los participantes mostraron previamente su voluntad expresa de participar en el estudio tras haberles sido debidamente explicado el procedimiento previsto y sus familias otorgaron consentimiento informado. Se respetaron escrupulosamente todos los principios éticos de la declaración de Helsinki en cuanto a anonimato y respeto de la integridad de los participantes.

\subsection{Instrumentos}

Se seleccionó la prueba PROLEC-R (Cuetos, Rodríguez, Ruano y Arribas, 2014) para determinar el nivel de desarrollo lector, ya que se trata de una prueba ampliamente utilizada en el entorno clínico de habla española y se caracteriza por tener en cuenta el modelo de la doble ruta de Coltheart y Rastle (1994), ya mencionado. Concretamente, se administraron las siguientes subpruebas: Nombre o Sonido de Letras, para evaluar el dominio de las correspondencias grafo-fonémicas, la de Lectura de Palabras, para evaluar el funcionamiento de la ruta léxica de lectura, la de Lectura de Pseudopalabras, para 
evaluar el funcionamiento de la ruta fonológica de lectura y la de Comprensión de Textos, para evaluar la eficacia de la lectura de los participantes. La prueba no dispone de baremos aplicables a la población panameña, por lo que se emplearon los obtenidos en su adaptación psicométrica para su uso con la población metropolitana de Lima (Cayhualla, Chilón y Espíritu, 2011; Cayhualla, Chilón y Espíritu, 2013), por ser esta más cercana cultural, lingüística y socioeconómicamente a la panameña que la obtenida en España (país de baremación original de la prueba).

Paralelamente, se utilizó la prueba WISC-IV (Wechsler, 2007) para evaluar el cociente intelectual de los participantes detectados con alteraciones de la lectura.

\subsection{Método}

Se empleó inicialmente la prueba PROLEC-R para determinar el nivel lector de todos los participantes en sesiones de evaluación individuales. Se seleccionó como posibles casos de DD a los participantes que presentaron rendimientos correspondientes a la categoría Dificultad Severa (al menos 2 desviaciones estándar por debajo del promedio) en el índice combinado (que une exactitud y velocidad de lectura) de la subprueba de Lectura de Palabras, para detectar casos con sospecha de dislexia superficial (puesto que esta subprueba penaliza, especialmente en el tiempo empleado, a los sujetos con escasa capacidad para reconocer la forma ortográfica de las palabras). Se empleó idéntico criterio con la subprueba de Lectura de Pseudopalabras para detectar los casos sospechosos de dislexia fonológica, puesto esta subprueba requiere del uso de la ruta fonológica de lectura, y con los que fracasaron en ambas subpruebas (sospechosos de dislexia mixta). También se retuvo como casos posibles a aquellos que presentaron niveles de desviación muy significativos (-2 desviaciones estándar en los baremos de la prueba) en comprensión de textos, presentando simultáneamente el nivel de Dificultad (-1 desviación estándar) tanto en la prueba de Lectura de Palabras como en la de Lectura de Pseudopalabras, en ausencia de trastorno del lenguaje oral. Aunque diversos autores y manuales diagnósticos emplean el criterio de 1.5 desviaciones estándar por debajo de la media, se prefirió emplear un criterio más estricto, aunque también de uso habitual.

Posteriormente, para cumplir con el criterio de exclusión de discapacidad intelectual se procedió a descartar como casos positivos a los participantes con cociente inferior a 70, criterio del manual DSMV (American Psychiatric Association, 2013), mediante la prueba WISC-IV.

\section{Resultados}

En el conjunto de la muestra $(n=216)$ fueron detectados 12 participantes $\left(6\right.$ en $4^{\circ}$ grado, 3 en $5^{\circ}$ grado y 9 en $6^{\circ}$ grado) que cumplieron con todos los criterios determinados, tras descartar la existencia de casos con posible explicación en el funcionamiento intelectual (se descartó para el cálculo de la prevalencia de DD a un participante con dificultad lectora severa que presentó simultáneamente un cociente intelectual inferior a 70).

Se empleó la siguiente fórmula para el cálculo de la prevalencia de dislexia del desarrollo:

$$
\text { Prevalencia }=\frac{\text { Casos }}{\text { Total de individuos }}
$$

por lo que la prevalencia total quedó establecida en $\mathbf{5 . 5 6 \%}\left(\frac{12}{216}\right)$. 


\section{Tabla 1}

Participantes detectados con DD, según género

\begin{tabular}{lll}
\hline Sexo & Positivos & $\mathbf{n}$ \\
\hline Niños & 6 & 108 \\
Niñas & 6 & 108 \\
Total & $\mathbf{1 2}$ & $\mathbf{2 1 6}$ \\
\hline
\end{tabular}

\section{Tabla 2}

Participantes detectados con DD, según tipo de dislexia

\begin{tabular}{ll}
\hline Subtipo Diagnóstico & Número de participantes detectados \\
\hline Dislexia Fonológica & 5 \\
Dislexia Superficial & 0 \\
Dislexia Mixta & 7 \\
Total & $\mathbf{1 2}$ \\
\hline
\end{tabular}

Como se puede observar en la tabla anterior, no se encontró ningún caso que tuviera únicamente dificultades severas en el uso de la ruta léxica de lectura, mientras que algunos participantes sólo presentaron dificultades severas en el uso de la ruta fonológica de lectura y una mayoría presentó alteraciones similares en ambas rutas de lectura.

\section{Tabla 3}

Distribución de los participantes detectados con DD, por tipo de escuela pública/particulary prevalencia

\begin{tabular}{llll}
\hline Grupos escuelas & Positivos & n & Prevalencia \\
\hline Públicas & 8 & 139 & $5.19 \%$ \\
Particulares & 4 & 77 & $5.76 \%$ \\
Total & $\mathbf{1 2}$ & $\mathbf{2 1 6}$ & $\mathbf{5 . 5 6 \%}$ \\
\hline
\end{tabular}

Dada la diversidad de métodos y criterios adoptados en los estudios de prevalencia efectuados en otros países, para hacer más descriptivo el estudio de la muestra se procedió complementariamente a calcular la prevalencia usando el criterio empleado en el trabajo llevado a cabo en Ciudad de Guatemala por Jiménez y García de la Cadena (2007). En dicho estudio, se consideró como casos positivos a los participantes que se encontraron por debajo de percentil 25 en precisión en lectura de pseudopalabras y simultáneamente estuvieron por encima del percentil 75 en tiempo empleado en lectura de palabras o de pseudopalabras (dentro de la muestra de cada grado). Para emplear esta metodología, que tiene la ventaja de no depender de baremos estandarizados localmente, se utilizaron en el presente trabajo los índices de precisión y velocidad de las subpruebas de Lectura de Pseudopalabras y de Lectura de 
Palabras. Con este procedimiento, se obtuvo un $\mathbf{1 4 . 3 5 \%}$ de prevalencia (31 participantes de los 216 , 12 en $4^{\circ}$ grado, 10 en $5^{\circ}$ grado y 9 en $6^{\circ}$ grado).

\section{Discusión}

La cifra de prevalencia de DD de 5.56\% obtenida en la Ciudad de Panamá se enmarca en el intervalo de valores esperados entre 3.2\% (Jiménez et al., 2009) y 11.8\% (Carrillo et al., 2011) de los estudios realizados en lengua española. Si atendemos a los referentes regionales cercanos, parece una cifra elevada comparada con las cifras de 3.3\% (De los Reyes et al., 2008) y 3.63\% (Pardo, 2019) obtenidas Colombia y con el 8\% de Ciudad de Guatemala (Jiménez y García de la Cadena, 2007), teniendo en cuenta que con similar metodología de detección obtendríamos en la muestra panameña un considerable $14.35 \%$ de prevalencia.

No se encontraron diferencias por género entre los casos, puesto que se distribuyeron al 50\% (en una muestra ya pareada por sexo). Este resultado es inesperado si lo relacionamos con la mayor parte de los trabajos epidemiológicos con tamaños de muestra más grandes, que determinan mayores tasas de prevalencia en varones (Lambe, 1999), aunque existen estudios recientes que aportan matices al tamaño de esta asimetría entre sexos (Arnett et al., 2017). En cualquier caso, el tamaño de muestra del presente estudio podría explicar que no se encontraran diferencias en este ámbito, como ocurre en otros estudios con muestras reducidas llevados a cabo en la región (Vélez et al., 2015).

Aunque no constituía el objetivo principal del estudio, se establecieron categorías diagnósticas entre los participantes con DD, siguiendo la clasificación clásica ya mencionada (dislexia del desarrollo fonológica, léxica, mixta), ampliamente utilizada por especialistas clínicos y por investigadores propuesta por Castles y Coltheart (1993). No se encontraron casos de daño severo exclusivo en la ruta léxica de lectura. En los casos en los que apareció daño severo en el uso de esta ruta, apareció simultáneamente en el uso de la fonológica, lo que sería coherente con el modelo de autoaprendizaje (Share, 1995), que postula que el uso repetido y correcto sobre una misma palabra de la ruta de descomposición grafo-fonémica permite que aquella termine incluyéndose en el léxico ortográfico visual, pasando a leerse por la ruta léxica, o directa. En base a la teoría de déficit fonológico como base del trastorno específico de la lectura, parece aceptarse que todas las personas con este trastorno presentan en mayor o en menor medida dificultades con el uso de la ruta fonológica de lectura (Carrillo, 2012), aunque en la lectura en español, por la transparencia de su ortografía, este tipo de dificultades tienda a atenuarse con el paso del tiempo. El uso tardío e ineficiente de la ruta fonológica de lectura podría provocar que el mecanismo de autoaprendizaje no se ejercite correctamente, lo cual explicaría la abundancia de perfiles mixtos hallados en el presente trabajo. No obstante, otros estudios más centrados en el objetivo de detectar tipos de dificultad lectora en español (Carrillo y Alegría, 2009; Cuadro y Marín, 2007) sí detectaron perfiles donde la alteración es más acusada en el uso de la ruta léxica que en el de la fonológica (disléxicos superficiales), lo que queda sin explicación en este trabajo. A pesar de ello, conviene considerar que el tamaño de la muestra obtenida (12 casos de DD) confiere escasa fortaleza a las conclusiones que se trate de establecer en cuanto a la distribución de los subtipos (los estudios mencionados trabajan directamente con muestras más amplias de casos ya diagnosticados previamente).

A pesar de que la prevalencia varió en las 6 escuelas en las que se llevó a cabo el estudio (lo cual es inevitable teniendo en cuenta el escaso tamaño de muestra que cada una de ellas aportaba), en todas ellas se detectaron casos. Es llamativo que no aparecieran diferencias estadísticamente significativas 
$(\mathrm{p} \leq 0.05)$ de prevalencia entre los estudiantes de escuelas públicas y de escuelas particulares $(5.19 \%$ contra 5.76\% respectivamente), con incluso una ligera ventaja por parte de los de las públicas. Esto parece corresponderse con el carácter neurobiológico del trastorno (Soriano-Ferrer y Piedra Martínez, 2017), que se presenta en todo tipo de entornos, aunque se pueda debatir si el factor ambiental (la estimulación recibida) podría contribuir a acentuar o a atenuar la severidad de los síntomas y, por lo tanto, las perturbaciones que provoca en el rendimiento escolar de quienes la padecen (Portellano, 2004).

\section{Conclusión}

Los resultados del presente trabajo parecen justificar la realización de un estudio de mayor envergadura (con un aumento significativo del tamaño de muestra y una aleatorización del muestreo con una estratificación proporcional por corregimientos) en el que se confirmen y amplíen los hallazgos. En cualquier caso, la importante tasa de prevalencia de dislexia encontrada pone en evidencia la necesidad otorgarle mayor relevancia el trastorno en Panamá e impulsar estrategias costo-efectivas para su abordaje mediante políticas públicas, dadas sus consecuencias escolares, laborales, psicológicas y sociales ya descritas en la literatura.

6. Financiación: El presente trabajo ha sido posible gracias a la financiación de la Universidad Católica Santa María la Antigua de Panamá.

\section{Referencias}

Alemany, C. (2019). Las consecuencias sociales de las dificultades de aprendizaje en niños y adolescentes Social consequences of learning difficulties in children and teenagers. Ehquidad International Welfare Policies and Social Work Journal, 11, 91-121. https://doi.org/10.15257/ehquidad.2019.0004

American Psychiatric Association, A. (2013). Diagnostic and statistical manual of mental disorders, (DSM$V)$. Arlington, VA: American Psychiatric Publishing.

Arnett, A. B., Pennington, B. F., Peterson, R. L., Willcutt, E. G., DeFries, J. C., \& Olson, R. K. (2017). Explaining the sex difference in dyslexia. Journal of Child Psychology and Psychiatry and Allied Disciplines, 58(6), 719-727. https://doi.org/10.1111/jcpp.12691

Carrillo, M.S., \& Alegría, J. (2009). Mecanismos de identificación de palabras en niños disléxicos en español: ¿Existen subtipos? Ciencias Psicológicas, 3(2).

Carrillo, M. S. (2012). La dislexia: bases teóricas para una práctica eficiente. Ciencias Psicológicas, VI(2), 185-194. https://doi.org/10.22235/cp.v6i1.75

Carrillo, M. S., Alegría, J., Miranda, P., \& Sánchez Pérez, N. (2011). Evaluación de la dislexia en la escuela primaria: Prevalencia en español. Escritos de Psicología, 4(2), 35-44.

https://doi.org/10.5231/psy.writ.2011.1407

Castles, A., \& Coltheart, M. (1993). Varieties of developmental dyslexia. Cognition, 47(2), 149-180. 
https://doi.org/10.1016/0010-0277(93)90003-E

Cayhualla, N., Chilón, D., \& Espíritu, R. (2011). Adaptación de la Batería de Evaluación de los Procesos Lectores Revisada PROLEC-R en estudiantes de Primaria de Lima Metropolitana (Pontificia Universidad Católica del Perú). Recuperado de http://tesis.pucp.edu.pe/repositorio/handle/20.500.12404/1309?show=full

Cayhualla, N., Chilón, D., \& Espíritu, R. (2013). Adaptación psicométrica de la Batería de Evaluación de los Procesos Lectores Revisada (PROLEC-R). Propósitos y Representaciones, 1(1), 39-57. https://doi.org/10.20511/pyr2013.v1n1.3

Coltheart, M., \& Rastle, K. (1994). Serial Processing in Reading Aloud : Evidence for Dual-Route Models of Reading. Journal of Experimental Psychology: Human Perception and Performance, 20(6), 1197-1211.

Cuadro, A., \& Marín, J. (2007). Subtipos de lectores retrasados en español. Ciencias Psicológicas, I(2), 133-148. https://doi.org/10.22235/cp.v0i1.545

Cuetos, F., Rodríguez, B., Ruano, E., \& Arribas, D. (2014). Test PROLEC-R. Batería de Evaluación de los Procesos Lectores, revisada (5 a edición). (TEA Edicio). Madrid: TEA Ediciones.

De los Reyes Aragón, C., Lewis Harb, S., Mendoza Rebolledo, C., Neira Meza, D., León Jacobus, A., \& Peña Ortiz, D. (2008). Estudio de prevalencia de dificultades de lectura en niños escolarizados de 7 años de Barranquilla (Colombia). Psicología Desde El Caribe, (22), 37-49.

Galaburda, A. M., Lo Turco, J., Ramus, F., Fitch, R. H., Rosen, G. D., \& Fisher Landau, E. (2006). La Dislexia del Desarrollo: Gen, Cerebro y Cognición. Psykhe (Santiago), 15(2), 3-11. https://doi.org/10.4067/S0718-22282006000200001

Jiménez E., J., Guzmán, R., Rodríguez, C., \& Artiles, C. (2009). Prevalencia de las dificultades específicas de aprendizaje: la dislexia en español. Anales de Psicología, 25(1).

Jiménez, J. E., \& García de la Cadena, C. (2007). Learning Disabilities in Guatemala and Spain: A Cross-National Study of the Prevalence and Cognitive Processes Associated with Reading and Spelling Disabilities. Learning Disabilities Research \& Practice, 22(3), 161-169. https://doi.org/10.1111/j.1540-5826.2007.00247.x

Lambe, E. K. (1999). Dyslexia, gender, and brain imaging. Neuropsychologia, 37(5), 521-536. https://doi.org/10.1016/S0028-3932(98)00146-8

Lyon, G. R.; Shaywitz, S. E.; Shaywitz, B. A. (2003). Defining Dyslexia , Comorbidity , Teachers ' Knowledge of Language and Reading A Definition of Dyslexia. Annals of Dyslexia, 53, 1-14.

Martin, A., Kronbichler, M., \& Richlan, F. (2016). Dyslexic brain activation abnormalities in deep and shallow orthographies: A meta-analysis of 28 functional neuroimaging studies. Human Brain Mapping, 37(7), 2676-2699. https://doi.org/10.1002/hbm.23202

Organización Mundial de la Salud (2018). Clasificación Internacional de Enfermedades undécima revisión (CIE-11). Ginebra: Organización Mundial de la Salud, OMS.

Pardo, A. N. (2015). Prevalencia del Trastorno Específico de la Lectura en una Muestra de Instituciones Educativas de la Localidad 19 de Bogotá. Biblioteca Digital UNAL, 130. 
Recuperado de http://www.bdigital.unal.edu.co/47004/1/05599185.2015.pdf

Portellano, J. A. (2004). La dislexia en todas las edades. Revista A Distancia, 22(2-3), 81-85.

Sainio, P. J., Eklund, K. M., Ahonen, T. P. S., \& Kiuru, N. H. (2019). The Role of Learning Difficulties in Adolescents' Academic Emotions and Academic Achievement. Journal of Learning Disabilities, 52(4), 287-298. https://doi.org/10.1177/0022219419841567

Serrano, F., \& Defior, S. (2004). Dislexia en Español: estado de la cuestión. Journal of Research in Educational Psychology, 2(2), 13-34. https://doi.org/10.25115/ejrep.v2i4.1148

Share, D. L. (1995). Phonological recoding and self-teaching : sine qua non of reading acquisition. Cognition, 55, 151-218.

Shaywitz, S. E. (1998). Dyslexia. New England Journal of Medicine, Vol. 338, pp. 307-312. https://doi.org/10.1056/NEJM199801293380507

Soriano-Ferrer, M., \& Piedra Martínez, E. (2017). Una revisión de las bases neurobiológicas de la dislexia en población adulta. Neurologia, Vol. 32, pp. 50-57. https://doi.org/10.1016/j.nrl.2014.08.003

Vélez, X., Tárraga, R., Fernández-Andrés, M. I., Sanz-Cervera, P., Blázquez-Garcés, J. V., \& Tijeras, A. (2015). Incidencia de la dislexia en Ecuador: Relación con el CI, lateralidad, sexo y tipo de escuela. International Journal of Developmental and Educational Psychology, 2(1), 249-257. https://doi.org/10.17060/ijodaep.2015.n1.v2.64

Wechsler, D. (2007). WISC-IV: Escala de Inteligencia de Wechsler para Niños-IV (2a ed.). Madrid: TEA Ediciones.

Wydell, T. N. (2012). Cross-Cultural/Linguistic Differences in the Prevalence of Developmental Dyslexia and the Hypothesis of Granularity and Transparency. In Dyslexia: A comprehensive and International approach (INTECH Ope, pp. 1-14). Croatia.

Zuppardo, L., Serrano, F., \& Pirrone, C. (2017). Delimitando el perfil emotivo-conductual en niños y adolescentes con dislexia. Revista Retos XXI, 1(1), 88-104. 